IL CALO DELLA PARTECIPAZIONE ELETTORALE

NELLE ELEZIONI AMMINISTRATIVE IN NORVEGIA

di TOR BJøRKLUND 
Ringrazio foannes Bergh per l'aiuto nella raccolta dei dati e fo Saglie per i commenti. 
1. Il declino della partecipazione elettorale: fenomeno diffuso e caso norvegese. Prospettive di analisi per le elezioni amministrative

Negli ultimi decenni le elezioni nei paesi occidentali hanno fatto registrare in molti casi bassi tassi di partecipazione. Nel 2001 il primo ministro inglese Tony Blair fu rieletto con un ampio margine di voti, ma la partecipazione dell'elettorato britannico non fu altrettanto impressionante: votò meno del $60 \%$ degli aventi diritto. Dobbiamo risalire al 1918 per registrare in Gran Bretagna un tasso di partecipazione più basso. Comunque la partecipazione elettorale continua a variare considerabilmente tra paesi e nel corso del tempo. Nel 2002 Chirac è stato rieletto presidente della repubblica francese con un tasso di partecipazione piuttosto alto $(81 \%)$, ma nel primo turno elettorale tale tasso era stato notevolmente più basso (72\%). La crescita della partecipazione al secondo turno è stata interpretata come l'effetto del discusso avversario di Chirac, Jean Marie Le Pen. Alcuni osservatori hanno infatti visto questa elezione come un referendum contro lo stesso Le Pen, che ha portato ad una mobilitazione alle urne in reazione alla sua candidatura.

Il dibattito sulla partecipazione elettorale riguarda di solito le elezioni nazionali, mentre le elezioni locali ne sono generalmente escluse e le relative statistiche non sono facilmente reperibili. Perciò è difficile determinare se c'è stato, nel tasso di partecipazione alle elezioni locali, un andamento decrescente analogo a quello della maggior parte delle elezioni nazionali (Topf 1995a; Rose 1997). Hoffmann-Martinot ha però osservato un andamento nelle elezioni locali simile a quello nelle nazionali: «Negli ultimi anni i cittadini delle democrazie occidentali stanno partecipando meno alle elezioni, elezioni locali comprese» (Hoffmann-Martinot 1994, p. 13).

Anche in Norvegia la partecipazione alle elezioni locali è andata nettamente declinando. Una partecipazione costantemente in calo può infatti essere osservata dall'inizio degli anni Sessanta alle ultime elezioni del 1999 (vedi FIG. 1). Fino agli anni Sessanta c'era stata invece una tendenza ascendente. Nella storia della partecipazione elettorale alle consultazioni locali in Norvegia dell'ultimo secolo si distinguono cioè due periodi: il primo dal 1901 al 1963, caratterizzato da una partecipazione crescente ed il secondo, dal 1963 al 1999, segnato invece da una 
partecipazione decrescente. Sempre in Norvegia, questi andamenti non sono stati altrettanto netti nelle elezioni politiche, nonostante nelle ultime due consultazioni la partecipazione vi sia decisamente diminuita.

Probabilmente in nessun altro paese dell'Europa occidentale si è avuto un continuo declino nella partecipazione alle elezioni amministrative come in Norvegia, sebbene l'andamento di lungo periodo tenda ad essere decrescente anche negli altri paesi. Il livello di partecipazione dell'elettorato norvegese è oggi molto meno alto rispetto al passato: da circa l' $80 \%$ del 1963 si è passati al 60\% del 1999. Ciò non toglie che sia ancora superiore a quello, per esempio, che si registra nelle elezioni locali in Gran Bretagna, che è del 40/45\%.

Il calo della partecipazione elettorale è un tema complesso. In questo saggio tenteremo di individuarne alcuni aspetti, relativamente alle elezioni amministrative norvegesi, concentrando l'analisi sulle sue cause e sulle sue conseguenze. Anticipiamone brevemente gli elementi essenziali.

Riguardo alle cause, il punto di partenza è costituito dai cambiamenti economici e sociali, anche se, alla luce delle trasformazioni avvenute negli ultimi decenni, sembrerebbe più facile menzionare fattori che dovrebbero incrementare il tasso di partecipazione elettorale piuttosto che farlo regredire: una scolarizzazione di massa, il varo di una televisione a diffusione nazionale nel 1973, il crescente numero di giornali, l'emergere cioè di una società dell'informazione con facile accesso alle fonti politiche attraverso i vari mass media e, ora, anche Internet.

Oltre a ciò, il ruolo politico dei 435 comuni norvegesi è cresciuto d'importanza. I comuni sono una pietra angolare dello stato sociale: essi funzionano come distributori e produttori di servizi sociali. Fin dagli anni sessanta il settore pubblico, in particolare a livello degli enti locali, ha conosciuto un'espansione continua. Circa il 20\% della forza lavoro è occupata nei comuni e nelle contee, la maggior parte nei settori del welfare. Ma i comuni non sono per i cittadini soltanto una parte dello stato sociale, essi hanno anche competenze di programmazione nell'edilizia e nello sviluppo economico. E tuttavia, nonostante questa accresciuta importanza politica del livello locale, vedremo che la partecipazione elettorale alle amministrative è crollata.

A questo proposito saranno osservati e discussi due andamenti opposti che sono stati definiti come «paradosso della partecipazione» (Baldersheim e Rose, 2000). Analizzando la partecipazione elettorale saranno inoltre introdotte due spiegazioni, una di carattere normativo ed una di carattere strumentale. Anticipiamole brevemente.

Da un punto di vista prettamente formale il voto è una questione giuridiconormativa e l'atto del votare un dovere civico, ossia il singolo cittadino, come membro di una società democratica, è obbligato a votare. In uno studio recentemente pubblicato è stato rilevato empiricamente l'impatto sul voto esercitato dal senso del dovere civico e dai benefici strumentali ottenibili. La conclusione di questa ricerca è stata: «Le considerazioni razionali sono molto meno importanti al momento del voto rispetto al senso del dovere: la percezione di avere un'obbliga- 
zione morale a votare sembra essere la motivazione predominante per andare alle urne» (Blais, Young e Lapp, 2000, p. 190). I nostri dati non ci consentono di misurare accuratamente il peso relativo del dovere civico rispetto ai benefici strumentali nel caso dell'elettorato norvegese, ma si può ragionevolmente supporre che la stessa conclusione possa essere applicata anche ad esso. La domanda che ci porremo è se il dovere civico come spiegazione della partecipazione elettorale abbia avuto sul voto un impatto costante nel corso del tempo o meno.

FIG. 1. - Tasso di partecipazione alle elezioni politiche e amministrative in Norvegia. 1945-1999.

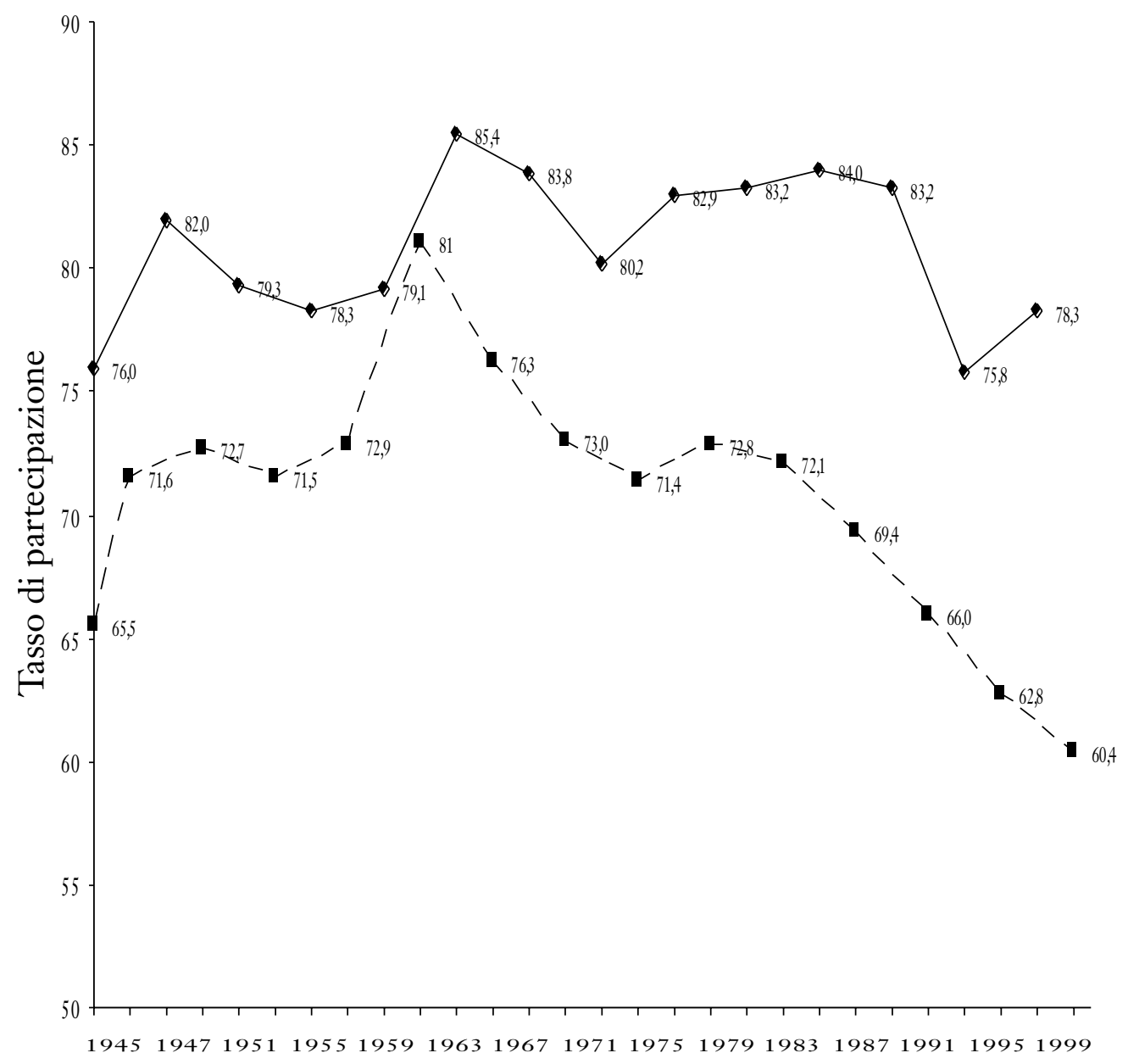

Anno

$\longrightarrow$ Elezioni politiche --- Elezioni amministrative

L'approccio normativo si contrappone ad una visione strumentale che, invece, mette al centro delle scelte politiche l'interesse personale e privato ed obiettivi egoistici (Downs, 1957). Questo secondo approccio, legato alle teorie della scelta 
razionale, interpreta il singolo voto come un atto irrazionale, poiché i costi dell'atto di votare spesso ne superano i benefici. Eppure si continua a votare. Da questa constatazione origina il cosiddetto "paradosso del voto".

Dal momento che in Norvegia il tasso di partecipazione è costantemente calato dal 1963 al 1999 la nostra ipotesi sarà che il peso del voto come dovere civico sia diminuito: ha perso vigore l'idea del votare come dovere del cittadino, a prescindere dall'importanza dell'elezione. Il dovere civico si presume venga interiorizzato in un processo di socializzazione secondaria e può dunque essere studiato in funzione delle "generazioni politiche". La nostra previsione sarà che le generazioni prebelliche hanno avuto un approccio strumentale al voto molto più marcato rispetto alle generazioni postbelliche.

Riguardo alle conseguenze della decrescente partecipazione elettorale, esse sono state ampiamente discusse in letteratura (Key Jr., 1958; Lijphart, 1997). Una delle argomentazioni più note afferma che un basso tasso di partecipazione mina la legittimità politica, dal momento che i rappresentanti eletti non possono parlare per conto della maggior parte dei votanti. Inoltre, un'ampia percentuale di astenuti è considerato un indicatore di diffusa sfiducia nei confronti dei politici locali. La sfiducia politica può tuttavia essere considerata sia come una causa, sia come una conseguenza della flessione nella partecipazione elettorale, ma, in ogni caso, i due fenomeni si suppone siano legati tra loro. Nel secondo caso a una partecipazione in calo dovrebbe seguire una flessione nella fiducia verso i politici. E questo il caso della Norvegia? Cercheremo di rispondere anche a questo interrogativo.

Inoltre, prenderemo in considerazione un'altra argomentazione sulle conseguenze del declino della partecipazione: una bassa partecipazione penalizza sistematicamente i partiti di sinistra poiché gli astenuti, nell'ipotesi che si recassero a votare, si suppone voterebbero a sinistra. La ragione è che i non votanti si trovano in proporzione maggiore tra gli appartenenti all'ultimo gradino della scala sociale, cioè tra le persone con predisposizione a sostenere quei partiti politici che favoriscono i non privilegiati. Ci si chiederà allora se la partecipazione calante ha davvero penalizzato in Norvegia i partiti schierati a sinistra, in particolare i laburisti.

Infine, discuteremo della partecipazione a movimenti monotematici. Questa forma di partecipazione politica ha mostrato, a differenza di quella elettorale, un andamento in crescita (Topf 1995b). Ciò fa emergere alcuni interrogativi: possiamo distinguere due diverse arene con differenti partecipanti, ossia un'arena a cui appartengono coloro che prendono parte, in varie forme, ad azioni politiche dirette ed un'arena unicamente elettorale? Secondo questa prospettiva sono soprattutto i non votanti ad essere coinvolti nella partecipazione a movimenti monotematici e quindi, dal momento che il numero dei non votanti è in crescita, anche il reclutamento in azioni politiche dirette dovrebbe aumentare.

L'ultima ipotesi che verrà testata empiricamente è quella che mette in relazione l'andamento del tasso di partecipazione con un cambiamento nelle norme e nei valori sociali. La diffusione di una visione strumentale della partecipazione politica legata ad interessi personali come forza trainante facilita o stimola la parteci- 
pazione monotematica. Di conseguenza questo (presunto) crescente numero di votanti che interpretano il voto in termini di interesse personale prenderà più frequentemente parte ad una partecipazione monotematica rispetto a coloro che percepiscono l'atto del voto come un dovere civico.

\section{Le cause}

Il contesto più generale del fenomeno del voto è legato al seguente interrogativo: perché alcuni vanno alle urne ed altri si astengono? La risposta a questa domanda dipende in modo stretto dall'ambito temporale di riferimento. Nel 1962 Stein Rokkan e Henry Valen pubblicarono un articolo sul comportamento elettorale in Norvegia (Rokkan e Valen, 1962) il cui focus, in modo non sorprendente, era sul crescente tasso di partecipazione, parzialmente spiegato da uno sviluppo graduale della periferia, cioè dalla sua progressiva mobilitazione. Fino allora la propensione al voto era stata particolarmente bassa tra le donne nella provincia, ossia nei comuni scarsamente popolati lontani dal centro, ma gradualmente anche queste periferie avevano cominciato ad essere mobilitate politicamente. Agli inizi degli anni sessanta la distanza tra centro e periferia stava per essere superata e le donne avevano quasi raggiunto il livello di partecipazione degli uomini. La propensione al voto continuava ad essere più o meno stabile nelle città, ma le statistiche elettorali mostravano un tasso di partecipazione sempre crescente, poiché c'erano sempre, nelle periferie, altri potenziali elettori, qualcuno che non aveva mai votato prima.

La mobilitazione della periferia fu considerata come il risultato di un processo di costruzione della nazione: essa era stata penetrata con più o meno successo dalle norme provenienti dal centro.

I partiti politici avevano cercato di creare delle loro articolazioni locali e gradualmente la provincia era stata connessa ad una rete di comunicazione, anche politica, a livello nazionale. L'ondata di partecipazione era quindi, secondo questa interpretazione, più o meno naturale. La metafora della costruzione della nazione riguardava perciò anche il tasso di partecipazione. E in questo caso i dati si accordavano con il modello teorico.

Subito dopo che Rokkan e Valen avevano pubblicato il loro articolo, però, l'andamento di lungo periodo dell'aumento del tasso di partecipazione elettorale si arrestò e cominciò una nuova fase. Quindi, dal momento che l'epoca dalla quale stiamo osservando il fenomeno è l'inizio del ventunesimo secolo, il nostro obiettivo non è, come lo era per Rokkan e Valen, tentare di spiegare perché la partecipazione elettorale stava aumentando, ma, al contrario, cercare di capire perché essa è costantemente in calo a partire dal 1963.

Per tentare di fare un po' di luce su questo tema tracciamo anzitutto un mappa di alcuni dei cambiamenti nella propensione al voto tra i diversi gruppi sociodemografici. 
Il processo di livellamento delle differenze nella propensione al voto tra centro e periferia, così come quello tra uomini e donne, è proseguito in Norvegia anche nel periodo di partecipazione elettorale decrescente. Inizialmente le differenze scomparvero e poi, almeno per quanto riguarda centro e periferia, si venne delineando una nuova tendenza in direzione opposta, come si vede dalla Fig. 2, dove è rappresentato graficamente il tasso di partecipazione elettorale dal 1945 al 1999 per i comuni più scarsamente e per quelli più densamente popolati ${ }^{1}$. In figura si osserva come, durante gli anni settanta, le differenze si siano appianate e come invece, a partire dagli anni ottanta, sia emersa una nuova tendenza destinata ad accentuarsi fino al 1999. Attualmente, cioè, la partecipazione elettorale più alta si registra in provincia.

FIG. 2. - Tasso di partecipazione alle elezioni amministrative norvegesi nei comuni densamente (più di 60.000 abitanti) ed in quelli scarsamente popolati (meno di 2.500 abitanti). 1945-1999.

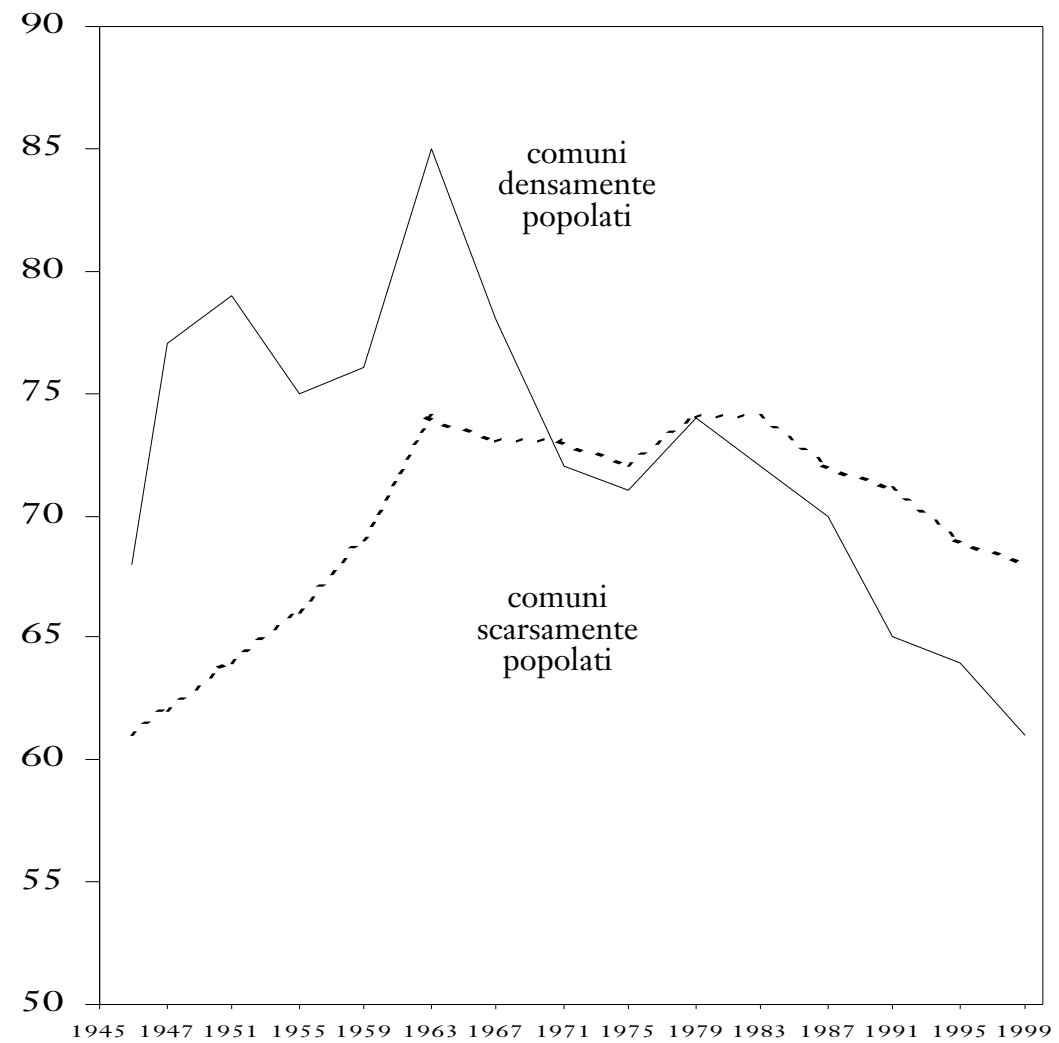

${ }^{1}$ I comuni scarsamente popolati sono quelli sotto i 2.500 abitanti, categoria che comprende 120 comuni; quelli densamente popolati sono i comuni sopra i 60.000 abitanti, categoria che comprende solo 7 comuni. 
Riguardo invece al rapporto tra sesso e tasso di partecipazione, l'elettorato femminile raggiunse il livello di quello maschile nel 1987. L'indicazione di una nuova tendenza è poi apparsa alle elezioni locali del 1995 quando le donne divennero l'elettorato più costante (vedi FIG. 3). Tuttavia, alle elezioni amministrative del 1999, di nuovo, non c'è stata differenza tra uomini e donne. Uno sguardo più attento ai dati rivela che ciò fu dovuto ad un alto tasso di partecipazione degli uomini anziani. Tra gli elettori sotto i 50 anni, invece, le donne hanno votato nettamente di più ${ }^{2}$. Se questa differenza può essere interpretata come il risultato di abitudini diverse nelle varie generazioni, si potrebbe prevedere che nel futuro le donne voteranno più spesso di quanto faranno gli uomini.

FIG. 3. - Tasso di partecipazione per sesso alle elezioni amministrative norvegesi dal 1945 al 1999.

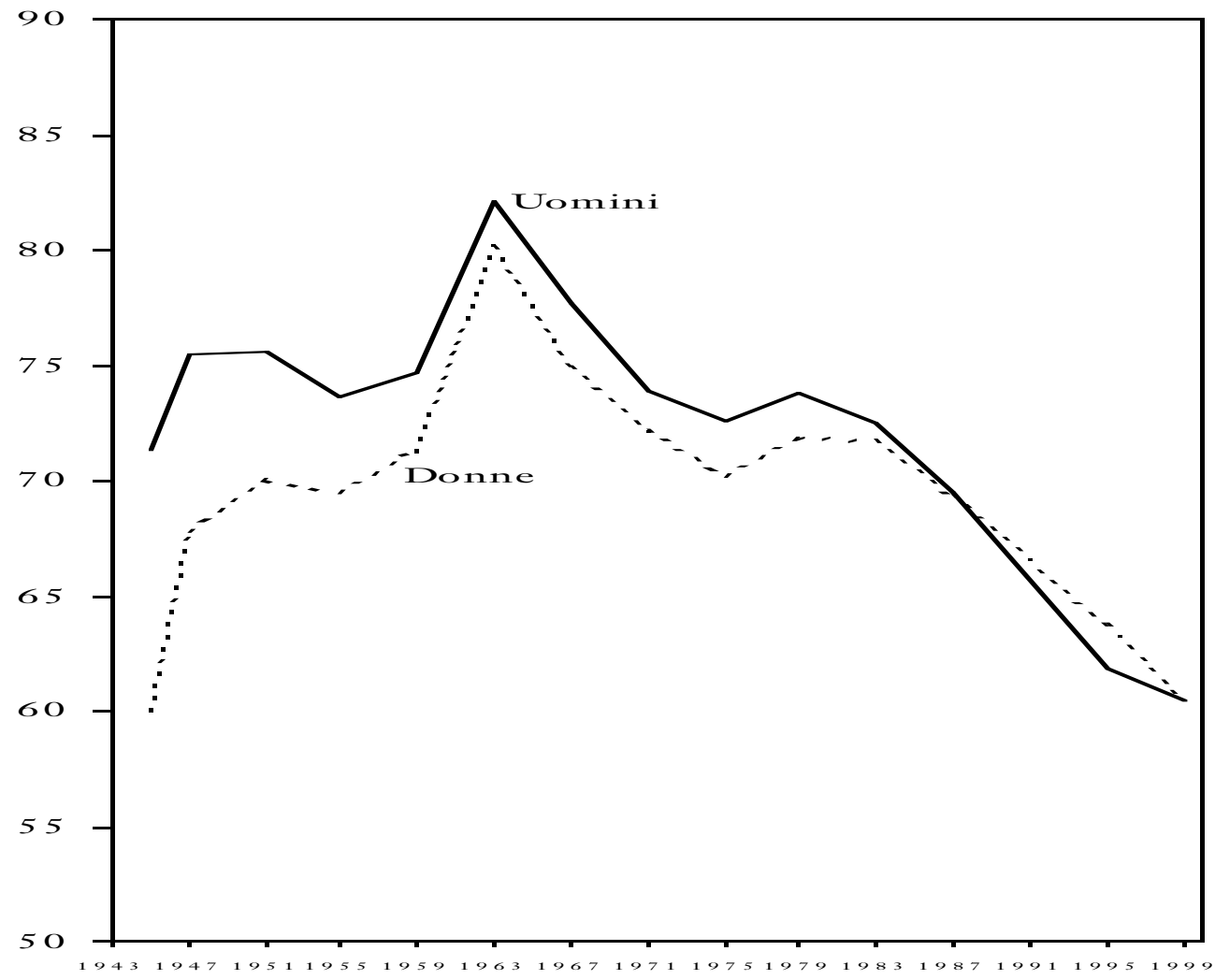

Fonti: Studi Elettorali Norvegesi per il periodo 1985-1993; Sondaggi Elezioni Amministrative per il 1995 ed il 1999.

\footnotetext{
${ }^{2}$ Votò il 57\% di donne contro il 50\% degli uomini.
} 
Ciò che risulta evidente, in ogni caso, è che i vecchi modelli sono cambiati. Gli uomini nelle grandi città erano considerati una volta come la categoria con la più alta propensione al voto e le donne della provincia come il gruppo con l'atteggiamento più passivo: i primi erano l'avanguardia, le seconde la retroguardia. Adesso il quadro sembra essere esattamente il contrario. Parlando per tratti generali, le donne nella provincia sembrano aver sorpassato gli uomini nelle città in quanto a propensione al voto ${ }^{3}$. Potremmo essere tentati di sostenere che i ritardatari sono divenuti l'avanguardia. Tuttavia ciò potrebbe essere in qualche modo deviante. Il livello di partecipazione tra le donne in periferia non è infatti aumentato ma è rimasto stabile o è leggermente diminuito.

Rokkan e Valen riassunsero il periodo precedente al 1963 con l'espressione «la mobilitazione della periferia». Il periodo seguente, dal 1963 al 1999, non può essere definito, si è visto, come un periodo di mobilitazione. Una sua caratterizzazione più appropriata potrebbe essere quella di "smobilitazione del centro" e "stabilizzazione della periferia". In effetti sembra che Rokkan e Valen avessero previsto proprio una smobilitazione del centro ${ }^{4}$.

Come abbiamo anticipato, per tentare di interpretare il calo del tasso di partecipazione elettorale alle elezioni amministrative norvegesi, faremo anzitutto riferimento alle spiegazioni basate sull'interesse personale e su una concezione utilitaristica e strumentale del voto $\mathrm{e}$, in seguito, a quelle basate sulle norme $\mathrm{o}$, più specificatamente, sull'interpretazione del voto come virtù civica, come obbligo del cittadino a votare.

Mark Franklin (1996) conferisce particolare importanza alla motivazione strumentale come fattore esplicativo del voto e del non voto. La questione centrale è stabilire se andare alle urne influisce o meno sul risultato elettorale finale. Presumibilmente, il tasso di partecipazione aumenterà se il risultato elettorale è in grado di determinare le politiche pubbliche. Un prerequisito per una relazione diretta tra esito elettorale ed attuazione delle politiche pubbliche è che le varie alternative a disposizione del potere esecutivo siano chiaramente diverse tra loro.

Secondo l'approccio strumentale il declino della partecipazione elettorale ha varie cause. Anzitutto, il crollo può essere spiegato dalla diminuita importanza delle elezioni, e quindi altri fattori, diversi dai risultati elettorali, possono decidere gli esiti delle politiche pubbliche. Oppure, il calo della partecipazione può dipendere dal fatto che le differenze tra le alternative proposte dai partiti politici sono consi-

${ }^{3}$ La differenza non è tuttavia così pronunciata. Inoltre, dal momento che dalle elezioni del 1987 in poi dobbiamo basarci su dati ricavati da sondaggi, la base empirica non è tanto solida. Comunque, alle elezioni del 1987 il tasso di partecipazione fu leggermente più alto tra le donne nei comuni al di sotto dei 2.500 abitanti che tra gli uomini nei comuni con oltre 60.000 abitanti (Bjørklund, 1999, p. 213).

$4 \ll \grave{E}$ paradossale che proprio in questo periodo di ritardata "politicizzazione" delle aree periferiche troviamo un certo numero di indicatori di crescente "depoliticizzazione" delle maggiori città: su questo sviluppo del centro ci riferiamo all'articolo di Ulf Torgersen su La tendenza verso il consenso politico» (Rokkan, 1970, p. 183). 
derate pressoché inesistenti. Secondo la teoria del "partito pigliatutto" di Kirchheimer (1966), infatti, i partiti politici diventano sempre più simili.

Vediamo come tutto ciò si applica al caso della Norvegia.

Per quanto riguarda i comuni norvegesi abbiamo già accennato che la loro importanza in termini di competenze e di ruolo nella sfera pubblica è cresciuta negli ultimi decenni. Ma nel nostro caso, ciò che è determinante non sono i poteri formali degli enti locali, bensì come gli elettori valutano l'importanza delle elezioni amministrative.

I dati mostrano, si è detto, che dal 1977 al 1999 il tasso di partecipazione elettorale è crollato dall' $81 \%$ al $64 \%$. Secondo l'approccio strumentale si presupporrebbe che anche la percezione di un impatto significativo degli esiti elettorali sulle politiche pubbliche sia altrettanto in calo. Dal momento che domande pressoché identiche sono state fatte in ricerche elettorali nel 1971, nel 1995 e nel 1999, questa relazione può essere testata empiricamente. Il risultato è che, nonostante il tasso di partecipazione elettorale sia calato in questi anni di 12,6 punti percentuali, le elezioni comunali sono invece percepite come più importanti nel 1999 che nel 1971 (vedi TAB. 1). La percentuale di elettori che ritiene che la consultazione elettorale abbia un elevato impatto «su ciò che avverrà nel comune nei prossimi quattro anni» è rimasta più o meno stabile ed intorno al $20 \%$. Si è invece registrato un incremento nella categoria "qualche impatto" ed un decremento in quella di coloro che ritengono non ci sia "nessun impatto" del voto sulle politiche attuate dal comune.

Riguardo al tasso di partecipazione delle varie categorie, il calo è particolarmente pronunciato tra coloro che ritengono che l'elezione abbia un " basso o nessuno" effetto su ciò che avverrà nel comune nei quattro anni seguenti. Nel 1971 era più frequente rilevare affermazioni del tipo «a prescindere dal fatto che le elezioni non abbiano importanza, io vado lo stesso a votare»: il $71 \%$ di coloro che appartenevano a questa categoria nel 1971 votavano, contro soltanto il 51\% nel 1999 . Ciò indica che l'idea del voto come obbligo civico ha perso terreno nel corso del tempo. Tuttavia, dal momento che domande sul dovere civico sono state poste soltanto alle elezioni del 1999, è difficile dare una risposta definitiva.

Dai dati a disposizione vediamo però che, anche nel 1999, ritenere il voto come dovere civico era ancora opinione diffusa. Dobbiamo ammettere che rilevare il sostegno ad una norma percepita generalmente come positiva è assai problematico 5 . Tuttavia, non tutti la avallano ed infatti, non sorprendentemente, si riscontra una netta differenza nella partecipazione al voto: tra coloro che sostengono il voto come interesse personale solo il $24 \%$ vota, contro il $64 \%$ dei fautori del dovere civico.

${ }^{5}$ È stata posta la seguente domanda: «Alcuni ritengono che votare sia un dovere civico, altri che si dovrebbe votare solo se si considera l'elezione importante per i propri interessi. Qual è la sua opinione? Lei lo considera un dovere per il cittadino o questi dovrebbe votare solo quando l'elezione diventa una questione di interesse personale?». L'87\% ha sostenuto l'alternativa del dovere civico contro il $13 \%$ che si è pronunciato a favore del voto come interesse personale. 
TАВ. 1. - Percezione dell'impatto delle elezioni amministrative sulla vita del comune e tasso di partecipazione elettorale. 1971, 1995 e 1999.

\begin{tabular}{lrrrrrrr}
\hline & \multicolumn{3}{c}{ Impatto elezioni ${ }^{1}$} & \multicolumn{2}{c}{$\begin{array}{c}\text { Partecipazione } \\
\text { elettorale }\end{array}$} & $\begin{array}{c}\text { Variazione } \\
\text { partecipazione 1999-1971 }\end{array}$ \\
\hline & 1971 & 1995 & 1999 & 1971 & 1995 & 1999 & \\
\hline Alto impatto & 20 & 23 & 21 & 86 & 75 & 73 & -13 \\
Medio impatto & 24 & 39 & 38 & 79 & 67 & 65 & -14 \\
Basso/nessun impatto & 56 & 38 & 41 & 71 & 54 & 51 & -20 \\
\hline Totale & 100 & 100 & 100 & & & & \\
$N$ & 2124 & 2792 & 3105 & & & & \\
\hline
\end{tabular}

Fonti: Per il 1971 sondaggio SSB; per il 1995 ed il 1999 Sondaggi Elezioni Amministrative.

${ }^{1}$ Nel 1971 era stata posta la seguente domanda: «Lei crede che il risultato elettorale di quest'autunno avrà un impatto alto, medio o basso/nullo su ciò che accadrà nel comune nei prossimi quattro anni?». Una domanda pressoché identica fu formulata nel 1995 nel sondaggio sulle elezioni amministrative: «Lei crede che il risultato delle elezioni amministrative avrà un impatto alto, medio o basso su ciò che accadrà nel comune nei prossimi quattro anni?». Nel 1999 la domanda fu posta nel modo seguente: «Lei crede che il risultato elettorale delle amministrative di quest'autunno avrà un impatto alto, medio o basso/nullo su ciò che accadrà nel comune nei prossimi quattro anni?».

${ }^{2}$ La categoria cha ha risposto «non so» era più ampia nel 1971 ed il suo tasso di partecipazione elettorale particolarmente basso.

L'idea del voto come interesse personale, cioè l'approccio strumentale, intende l'atto di votare come una forma di calcolo relazionato all'importanza e alla rilevanza dell'elezione. Seguendo quest'approccio ci si aspetta allora che il tasso di partecipazione varii nettamente tra coloro che considerano l'elezione importante e coloro che, invece, la ritengono più o meno irrilevante. In altre parole, se l'elezione in questione è considerata poco importante, ci si attende che il tasso di partecipazione sia particolarmente basso tra i sostenitori dell'opzione dell'interesse personale. Coloro che, invece, ritengono il voto un dovere civico, proprio per questo andranno presumibilmente a votare a prescindere dall'importanza dell'elezione. Nella TAB. 2 questi risultati attesi sono in un certo senso confermati. Il fatto di andare a votare o meno dipende molto di più da una valutazione dell'importanza dell'elezione per un elettore che sostiene l'alternativa dell'interesse personale piuttosto che per uno che ritiene il voto un dovere civico.

Nella ricerca sulle elezioni del 1971 non possiamo distinguere tra "dovere civico" e "interesse personale", ma la differenza nel tasso di partecipazione tra coloro che ritengono alto l'impatto dell'elezione e coloro che, invece, lo considerano basso, non è così pronunciato (15 punti percentuali, vedi ancora TAB. 1). Questa distribuzione, tuttavia, non supporta molto l'approccio strumentale del voto. Siamo pertanto propensi a pensare che il voto come forma di dovere civico fosse un fattore centrale nel 1971. La distribuzione del 1999, invece, si accorda di più con l'approccio strumentale di quanto non faccia quella delle elezioni amministrative del 1971, dal momento che la differenza nella partecipazione elettorale sale a 22 
punti percentuali ${ }^{6}$ tra coloro che stimano alto l'impatto delle elezioni e coloro che, invece, lo ritengono basso o nullo.

ТАВ. 2. - Percezione dell'impatto delle elezioni amministrative sulla vita del comune e tasso di partecipazione elettorale a seconda che il voto sia considerato come dovere civico o come interesse personale. Valori percentuali ed assoluti. Elezioni amministrative 1999.

\begin{tabular}{lccccc}
\hline Impatto delle elezioni & \multicolumn{2}{c}{ Partecipazione elettorale } & \multicolumn{2}{c}{ N } \\
& & Dovere civico $^{1}$ & Interesse personale & Dovere civico & Interesse personale \\
\hline Alto impatto & 21 & 75 & 52 & 543 & 48 \\
Medio impatto & 38 & 71 & 28 & 995 & 156 \\
$\begin{array}{l}\text { Basso / nessun } \\
\text { impatto }\end{array}$ & 41 & 61 & 16 & 1041 & 238 \\
\hline $\begin{array}{l}\text { Totale/ media } \\
N\end{array}$ & 100 & 68 & 24 & 2579 & 442 \\
\hline
\end{tabular}

Fonte: Sondaggi Elezioni Amministrative 1999.

${ }^{1}$ Rilevato con la seguente domanda: «Alcuni ritengono che votare sia un dovere civico, altri che si dovrebbe votare solo se si considera l'elezione importante per i propri interessi. Qual è la sua opinione? Lei lo considera un dovere per il cittadino o questi dovrebbe votare solo quando l'elezione diventa una questione di interesse personale?».

Il dovere civico può essere considerato come un valore assimilato durante un processo di socializzazione che prescinde dalle epoche storiche. Ci si può comunque attendere che il livello di sostegno a tale valore varii tra le differenti generazioni. Dal momento che la domanda sul dovere civico è stata posta solo nel 1999, un'analisi per coorti risulta impossibile anche se il campione può essere suddiviso ugualmente in varie generazioni. Nella TAB. 3 sono riportati, per coorti, il tasso di partecipazione elettorale e la percentuale di coloro che affermano che votare sia una questione di interesse personale.

TAB. 3. - Motivazione utilitaristica del voto e tasso di partecipazione per coorti. Valori percentuali ed assoluti. Elezioni amministrative 1999.

\begin{tabular}{lcccc}
\hline \multicolumn{1}{c}{ Classe di età } & $\begin{array}{c}\text { Approccio } \\
\text { utilitaristico al voto }\end{array}$ & $\begin{array}{c}\text { Partecipazione } \\
\text { elettorale }\end{array}$ & N \\
\hline Dopo il 1975 & 29 & 31 & 272 & 427 \\
$1966-1975$ & 19 & 47 & 523 & 744 \\
$1956-1965$ & 10 & 63 & 608 & 822 \\
$1946-1955$ & 13 & 68 & 654 & 866 \\
$1930-1945$ & 6 & 72 & 723 & 971 \\
$1920-1929$ & 5 & 63 & 340 & 469 \\
\hline Totale & 12 & 60 & 3120 & 4299 \\
\hline
\end{tabular}

Fonte: Sondaggi Elezioni Amministrative 1999.

${ }^{6}$ Secondo la Tabella 1, nel 1999, la differenza è tra il 73\% (alto impatto) ed il 51\% (basso/nessuno impatto). I valori corrispondenti nel 1971 erano, rispettivamente, dell' $86 \%$ e del $71 \%$. 
L'andamento è chiaro: maggiore è la percentuale degli elettori, in una certa coorte, che ritengono il voto legato all'interesse personale, minore è il tasso di partecipazione elettorale nella coorte stessa. La variazione di tale tasso in funzione della categoria generazionale è pronunciato e saremmo tentati di suggerire che il declino, nelle generazioni più giovani, dell'idea del voto come dovere civico sia una causa del basso tasso di partecipazione generale.

Si può presumere pertanto che il voto come dovere civico avesse maggiori consensi in periodi precedenti, cioè quando le attuali generazioni più giovani, nelle quali i sostenitori dell'interesse personale sono più frequenti, non facevano ovviamente parte dell'elettorato. Di conseguenza, si può presumere anche che la variazione nella partecipazione in funzione della generazione non sia stata in passato così netta come lo è stata alle elezioni del 1999.

Nella TAB. 4 è riportato il tasso di partecipazione elettorale alle elezioni amministrative del 1971, 1995 e 1999, sempre per coorti. Vi viene confermato un modello assai noto: il tasso di partecipazione dipende dal ciclo di vita (Rosenstone e Hansen, 1993), ossia la partecipazione aumenta quanto più l'elettorato invecchia. La ragione è semplice: progressivamente, al momento di lasciare la scuola e le università per cercare un lavoro, crearsi una famiglia, ecc..., gli elettori diventano sempre più integrati nella società.

Questi elettori "integrati” si recano alle urne più spesso di quanto non facciano gli elettori meno integrati. All'altro estremo, ed in modo analogo, quando si considerano classi con età più avanzate, il tasso di partecipazione elettorale scende. Il pensionamento e la scomparsa delle reti sociali esistenti fino a quel momento contribuiscono a tale declino.

ТАВ. 4. - Tasso di partecipazione alle elezioni amministrative norvegesi per coorti. 19711999. Valori percentuali ed assoluti.

\begin{tabular}{lrrrrrr}
\hline \multicolumn{1}{c}{ Classe di età } & 1971 & 1995 & 1999 & \multicolumn{3}{c}{ N } \\
\hline Dopo il 1975 & - & 46 & 31 & - & 112 & 427 \\
$1966-1975$ & - & 42 & 47 & - & 597 & 744 \\
$1956-1965$ & - & 65 & 63 & - & 586 & 822 \\
$1946-1955^{1}$ & 53 & 67 & 68 & 254 & 586 & 866 \\
$1930-1945$ & 72 & 73 & 72 & 731 & 568 & 971 \\
$1920-1929$ & 76 & 75 & 63 & 509 & 328 & 469 \\
$1910-1919^{2}$ & 82 & 74 & - & 477 & 77 & - \\
Prima del 1910 & 74 & - & & 564 & - & - \\
\hline Totale & 73 & 63 & 60 & 2535 & 2854 & 4299 \\
\hline
\end{tabular}

Fonte: Sondaggi Elezioni Amministrative 1999.

${ }^{1}$ Nella ricerca del 1971 gli intervistati più giovani erano nati nel 1951.

${ }^{2}$ Nella ricerca del 1995 gli intervistati più anziani erano nati nel 1915. 
Pertanto, è possibile fornire un'ulteriore spiegazione del tasso di partecipazione elettorale decrescente facendo riferimento ad un'ipotesi generazionale. La differenza nella partecipazione tra le generazioni più giovani e quelle più vecchie aumenta dal 1971 al 1995 e lo stesso avviene spostandoci dal 1995 al 1999. Di conseguenza si sono evidenziati in modo più netto profili elettorali diversi in funzione della generazione. Ciò è dovuto, almeno in parte, ad un tasso di partecipazione particolarmente basso tra i giovani entrati a far parte dell'elettorato. Nelle classi nate dopo il 1966, tale tasso non ha mai superato il 50\%. La partecipazione alle elezioni locali del 1999 fu bassa come non mai tra i nati dopo il 1975, pari a soltanto al 31\%. Perciò il calo di partecipazione elettorale può essere attribuito a due fattori: un effetto-periodo che investe tutte le classi di età ed un effetto-generazione per il quale i più giovani votano meno di quanto facessero prima.

Warren E. Miller (1992) sottolinea, in un'analisi del declino del tasso di partecipazione elettorale negli Stati Uniti dal 1950 al 1980, l'importanza della generazione politica, intendendo per generazione un gruppo composto da individui con esperienze formative comuni. Con il passare del tempo la composizione generazionale dell'elettorato cambia. L'inclusione nell'elettorato delle generazioni postbelliche, o di quella che è stata chiamata la generazione post-New Deal, ha contribuito al crollo della partecipazione elettorale statunitense. La ragione è che queste nuove generazioni hanno una propensione al voto sistematicamente più bassa rispetto alla generazione del New Deal, che aveva vissuto la Grande Depressione negli anni della sua formazione. Il declino della partecipazione può perciò essere parzialmente spiegato, anche in Norvegia, dal fatto che la quota dell'elettorato appartenente alle generazioni successive al periodo del New Deal sta aumentando e che, dall'altro lato, sta invece scomparendo gradualmente quella che l'epoca del New Deal l'aveva vissuta.

\section{Le conseguenze}

Nell'introduzione abbiamo accennato a due possibili conseguenze di una partecipazione elettorale in declino: in primo luogo, il basso tasso di partecipazione mina la legittimità politica del sistema e, in secondo luogo, penalizza sistematicamente i partiti di sinistra. Resta da chiedersi come tali possibili conseguenze valgano per le elezioni locali in Norvegia.

Riguardo alla prima conseguenza, cioè alla legittimità del sistema politico e alla fiducia nei politici, l'elettorato norvegese è generalmente classificato ad un livello piuttosto alto rispetto a quello di altri paesi (Aardal, 1999). Sulla questione della "fiducia nel Parlamento", ad esempio, il World Value Survey, ha classificato i norvegesi al primo posto in Europa con il più alto livello di fiducia (Listhaug e Wiberg, 1995, pp. 304-305). Se dunque ci fosse stata in Norvegia una relazione, seppur minima, tra partecipazione elettorale e grado di fiducia politica, quest'ultimo avrebbe dovuto crollare drammaticamente tra il 1963 ed il 1999. Ma così non è stato. 
Riguardo invece all'effetto dei risultati elettorali, dobbiamo chiederci quali partiti sono penalizzati da un'alta astensione elettorale. Arend Lijphart (1997) sottolinea, con una tesi peraltro ben corroborata, come la partecipazione elettorale sia legata alla condizione socio-economica. I non votanti appartengono in proporzione maggiore a categorie con livello di istruzione elementare, a basso reddito e a modesta condizione occupazionale. Questa composizione di classe che caratterizza i non votanti ha anche un impatto politico: una partecipazione elettorale diseguale può significare un'influenza politica diseguale. Non partecipando, o partecipando meno, certe classi sociali hanno meno possibilità di influenzare la sfera politica. Inoltre, come evidenzia V.Key (1958), i non votanti non costituiscono una categoria elettorale particolarmente interessante per i politici. Una sorta di circolo vizioso potrebbe quindi emergere, dal momento che gli astenuti diventano alienati dal processo politico: essi sono dei fuoriusciti ed in seguito, dal momento che i politici non tengono conto dei loro interessi, potrebbe essere difficoltoso reintegrarli.

In linea con quest'argomento si presume che i partiti di sinistra raccolgano una quota più alta di voti nei paesi con alto tasso di partecipazione, dal momento che questi partiti normalmente raccolgono elettori all'estremo più basso della scala sociale. Questa relazione empirica non è stata pienamente confermata. Tuttavia, uno studio sulle elezioni nazionali in 19 democrazie industrializzate tra il 1950 ed il 1990, sembrerebbe confermare una tendenza in questa direzione (Pacek e Radcliff, 1995).

Per quanto riguarda la Norvegia l'impatto del non voto sulla forza dei singoli partiti politici non è stata esaurientemente studiata. Ad una prima osservazione sembrerebbe essere plausibile l'ipotesi che la posizione del partito laburista sia stata penalizzata dal calo della partecipazione. Alle elezioni locali del 1963, quando il tasso di partecipazione fu dell' $81 \%$, i laburisti ottennero il 45,8\% dei voti. Nel 1999 i dati corrispondenti sono stati del $64 \%$ e del $28,6 \%$. Tuttavia, l'argomento per cui la perdita di consenso dei laburisti sia dovuta al declino della partecipazione elettorale pare essere troppo semplicistica. Il sostegno al partito laburista è diminuito anche alle elezioni politiche, nonostante il fatto che in queste il tasso di partecipazione non sia variato molto negli ultimi decenni.

Tentativi di mettere in relazione il crollo della partecipazione alle elezioni amministrative tra il 1995 ed il 1999 con la posizione elettorale del partito laburista sono stati fatti (Bjørklund e Saglie, 2000), ma nei risultati non si è riscontrata alcuna evidenza a favore dell'ipotesi secondo cui i laburisti debbano aver risentito più degli altri partiti di un alto numero di astenuti tra i loro elettori. Va comunque sottolineato che, in questo caso, l'evidenza è in qualche modo limitata, poiché i dati coprono soltanto il periodo tra le ultime due elezioni. Tentiamo, comunque, di guardare al problema in una prospettiva di lungo periodo.

L'argomento per cui i partiti di sinistra hanno perso voti tra il 1963 ed il 1999 a causa del calo della partecipazione elettorale è basato sull'assunto, precedentemente illustrato, secondo cui gli astenuti apparterrebbero soprattutto alle classi sociali disagiate. Poiché, tradizionalmente, i votanti in queste categorie sono 
predisposti a votare a sinistra, la conseguenza sarà una penalizzazione, nel caso norvegese, del partito laburista. Si tratta in ogni caso di una questione da risolvere empiricamente: il calo della partecipazione ha effettivamente determinato una diversa composizione dell'elettorato, riducendo l'ampiezza di certe categorie socioeconomiche di elettori che si astengono di più? Ed inoltre, questa distorsione si accentua al diminuire della quota di elettorato votante?

Tra il 1971 ed il 1995 la partecipazione elettorale in Norvegia si è ridotta di 10 punti percentuali. Secondo due indicatori socio-economici (reddito e livello di istruzione) i cambiamenti concomitanti nella composizione dell'elettorato sono stati limitati. Anzi, tali cambiamenti tendevano a mostrare qualche diminuzione nelle differenze sociali tra votanti e non votanti (Bjørklund, 1999). Comunque i dati sono parzialmente contraddittori.

Il crollo del tasso di partecipazione dal 1971 al 1999 è stato particolarmente pronunciato tra i colletti blu (Bjørklund e Saglie, 2000). Questa conclusione risulterebbe in linea con quelle tratte da Jørgen Goul Andersen e Jens Hoff (2000) che evidenziano come il declino della partecipazione in Scandinavia possa essere spiegato con la smobilitazione di classe. L'evoluzione della partecipazione dal 1971 al 1999 fornisce perciò delle indicazioni, anche se non prive di contraddizioni, che il declino della partecipazione ha aumentato il disequilibrio nella composizione di classe dell'elettorato. Questo sempre assumendo, come abbiamo esposto precedentemente, che i non votanti appartengano molto di più ai gradini inferiori della scala sociale che non agli altri. Se questo è vero, diminuendo la partecipazione soprattutto tra le classi sociali disagiate, queste risultano sottorappresentate nella composizione dell'elettorato votante norvegese. Dobbiamo inoltre ricordare che, negli ultimi decenni, le classi sociali più basse, in Norvegia, non votano più soltanto il Labour. Assumendo perciò che nello stesso periodo la composizione sociale dei votanti nei confronti dei non votanti sia rimasta più o meno costante, possiamo evincerne che, o alcuni partiti politici hanno abbassato il profilo sociale del loro elettorato rispetto al passato, o il varo di nuovi partiti (come il Partito del progresso) hanno attratto votanti nella parte bassa della scala sociale.

Se tutto ciò è vero si può concludere, in relazione alla composizione sociale dell'elettorato, che il calo della partecipazione ha, in effetti, penalizzato soprattutto i laburisti. In ogni casi questo è solo un tentativo di generalizzazione, dal momento che in questo campo manca ancora molto lavoro empirico.

\section{Tasso di partecipazione elettorale e partecipazione politica monotematica}

In un saggio sulla partecipazione politica Robert Topf conclude che «laddove il tasso di partecipazione elettorale nei paesi dell'Europa occidentale è rimasto notevolmente stabile, la partecipazione politica aldilà del voto è andata crescendo drammaticamente» (Topf, 1995b, p. 52). In realtà, in Norvegia, come abbiamo visto, la partecipazione elettorale alle elezioni amministrative non è stata stabile. Ma anche in Norvegia «la partecipazione politica aldilà del voto» (Bjørklund e Saglie, 
2000, p. 86) è chiaramente aumentata. Questa forma di impegno è spesso definita "non convenzionale" per distinguerla da quella convenzionale come, appunto, la partecipazione elettorale. Il termine "non convenzionale" può essere frainteso dal momento che, invece, questa forma di partecipazione si è assai diffusa. Soprattutto si incorre in un'associazione errata quando il termine "non convenzionale" viene considerato sinonimo di "anormale". Invece esso indica tutti quei tipi di partecipazione che non sono rivolti ai canali della rappresentanza (partiti ed organizzazioni), ma tentano di influenzare i decisori politici attraverso petizioni, manifestazioni, azioni politiche, ecc... La partecipazione non convenzionale si concentra principalmente su temi specifici ed il termine più appropriato per definirla potrebbe perciò essere partecipazione monotematica (Goul Andersen e Hoff, 2000).

In Norvegia ad un declino di lungo periodo del tasso di partecipazione elettorale si affianca un aumento, sempre nel lungo periodo, della partecipazione monotematica. Dati questi due andamenti opposti possiamo interrogarci sull'esistenza di due arene politiche diverse, caratterizzate da attori politici diversi. Ciò presupporrebbe che i votanti siano soggetti diversi rispetto a coloro che partecipano ad azioni politiche dirette. La ricerca empirica ha tuttavia mostrato che questa supposizione è completamente errata (Olsen e Sætren, 1980). I partecipanti più attivi nelle azioni monotematiche sono, anzi, coloro che votano e che sono iscritti a partiti politici. Data però la recente diffusione della partecipazione monotematica, negli ultimi anni la differenza tra votanti e non votanti in questo tipo di partecipazione è diminuita. In altre parole l'incremento della partecipazione monotematica è stato più pronunciato tra i non votanti e coloro che non sono iscritti a partiti politici ${ }^{7}$.

Due forme di azione politica diretta, indicatori di partecipazione monotematica, saranno qui prese in considerazione: firmare petizioni e partecipare a manifestazioni ${ }^{8}$. Come mostrato dalla figura 4 , si osserva un andamento ascendente per entrambi i tipi di attività e specialmente per quella riguardante la firma di petizioni. Un indice additivo è stato costruito per queste due attività. Sulla base del

${ }^{7}$ Ciò può essere esemplificato facendo riferimento ai dati sulla frequenza con cui si firma una petizione. Nel 1985 il 19\% aveva firmato una petizione contro il 30\% del 1999. Tra coloro che avevano votato alle elezioni, tra l' 85 ed il '99 i firmatari sono passati dal $20 \%$ al $31 \%$ e tra i non votanti dal $15 \%$ al $28 \%$. I valori corrispondenti tra gli iscritti sono stati, rispettivamente, del $30 \%$ e del $35 \%$ e tra i non iscritti del 17\% e del 30\% (Bjørklund e Saglie, 2000).

${ }^{8} \mathrm{Ci}$ si è basati sulla seguente domanda: $« \mathrm{Ci}$ sono diversi modi di esercitare un'influenza politica. Lei ha provato, nel corso degli ultimi quattro anni, ad influenzare una decisione concernente una questione specifica in consiglio comunale o nel consiglio di contea: a) firmando una petizione, b) partecipando ad un'azione di gruppo, ad un incontro di protesta o ad una manifestazione?». Questa domanda è stata inizialmente posta negli studi elettorali nazionali, relativamente alle elezioni del Parlamento monocamerale norvegese, lo Storting, ma in modo parzialmente diverso. Lo scopo della partecipazione non si limitava soltanto ad «una decisione concernente una questione specifica in consiglio comunale o nel consiglio di contea», ma includeva tutti i tipi, non ben specificati, di aree decisionali «nel corso degli ultimi quattro anni». Nonostante le domande poste alle elezioni amministrative siano più limitate rispetto alle corrispondenti poste per le elezioni dello Storting, esse hanno permesso di rilevare un maggior livello di partecipazione politica. 
punteggio medio dell'indice ${ }^{9}$ i votanti sono stati un po' più attivi dei non votanti (vedi tabella 5). Il grado di impegno, tuttavia, varia in modo significativo in funzione delle classi di età. Tra i nati dopo il 1975 due dati vanno registrati: una partecipazione monotematica eccezionalmente alta ed una partecipazione elettorale estremamente bassa. Inoltre, questa categoria è l'unica in cui la partecipazione monotematica è più alta, anche se non statisticamente rilevante, tra i non votanti che tra i votanti. Nelle altre classi di età, ad eccezione della seconda coorte più giovane (i nati tra il 1966 ed il 1975), la differenza tra votanti e non votanti è marcata.

FIG. 4. - Elettori che hanno firmato petizioni o partecipano a manifestazioni dal 1985 al 1999. Valori percentuali.

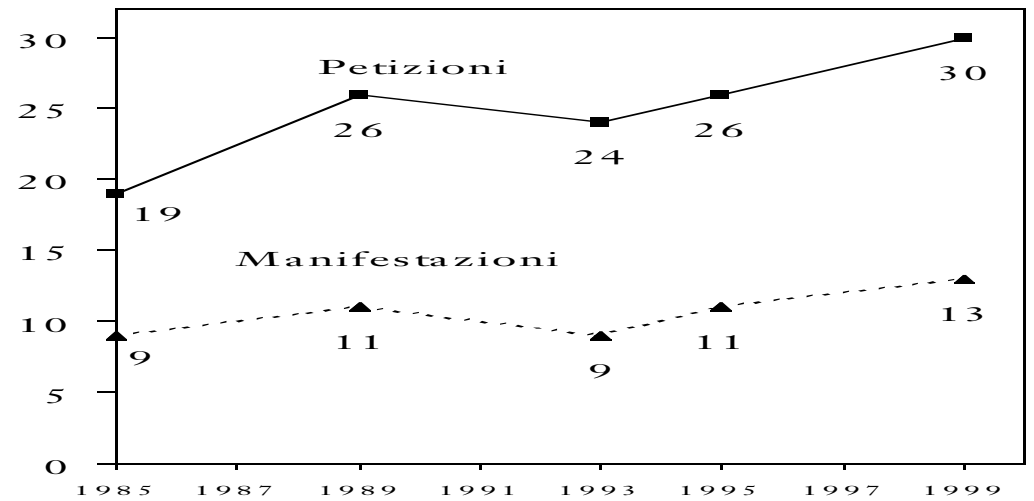

Fonti: Studi Elettorali Norvegesi per il periodo 1985-1993; Sondaggi Elezioni Amministrative per il 1995 ed il 1999.

Riguardo al livello di partecipazione politica non elettorale, inoltre, una linea di demarcazione può essere tracciata tra le generazioni postbelliche, cioè i nati dopo il 1945, e quelle precedenti (si veda ancora una volta la TAB. 5). Un'azione politica diretta e quindi, nella nostra accezione, una partecipazione monotematica, risulta essere più diffusa tra le generazioni postbelliche, cioè quelle che hanno ottenuto il diritto di voto a partire dal 1965 , periodo, come sappiamo, con un tasso di partecipazione elettorale calante. Saremmo allora tentati di dare una spiegazione generazionale sia all'incremento della partecipazione monotematica che al declino di quella elettorale.

Dal momento, cioè, che le generazioni postbelliche formano con il tempo quote sempre più ampie dell'elettorato complessivo, al contrario delle generazioni prebelliche che invece si riducono, questo avvicendamento potrebbe spiegare sia l'andamento crescente nella partecipazione monotematica che quello, invece, decrescente nell'affluenza alle urne che si osservano dai dati raccolti.

${ }^{9}$ L'indice va da 0 a 2 distinguendo coloro che hanno, sia partecipato a manifestazioni che firmato petizioni (2), da coloro che sono stati inattivi (0) e da coloro che hanno partecipato ad una delle due forme di azione (1). 
ТАВ. 5. - Punteggio medio, per generazione ed in funzione del voto/non voto, su un indice di partecipazione politica basato sulla presenza a manifestazioni elo sulla firma di petizioni.

\begin{tabular}{lrrrrrr}
\hline \multicolumn{1}{c}{ Classe di età } & Media & Votanti & Non votanti & \multicolumn{2}{c}{ N } \\
\hline Dopo il 1975 & 0,59 & 0,57 & 0,61 & 280 & 125 & 154 \\
$1966-1975$ & 0,48 & 0,51 & 0,44 & 530 & 314 & 216 \\
$1956-1965$ & 0,55 & 0,60 & 0,40 & 613 & 465 & 148 \\
$1946-1955$ & 0,46 & 0,49 & 0,33 & 660 & 523 & 138 \\
$1930-1945$ & 0,32 & 0,34 & 0,22 & 742 & 613 & 128 \\
$1920-1929$ & 0,22 & 0,26 & 0,06 & 352 & 280 & 72 \\
\hline Media & 0,43 & 0,45 & 0,38 & 3177 & 2320 & 857 \\
\hline
\end{tabular}

Fonte: Sondaggi Elezioni Amministrative 1999.

Il voto e la partecipazione politica monotematica possono essere analizzati anche in funzione del loro rapporto con differenti idee e valori. Si può allora supporre che i gruppi di azione incentrati su temi specifici attraggano di più gli elettori che hanno un approccio strumentale all'attività politica. A differenza dei gruppi di azione e dei movimenti, i partiti politici sono maggiormente costretti a bilanciare gli interessi e ad avere una visione complessiva della società. Per i gruppi di azione monotematica la piattaforma di mobilitazione può invece, con più facilità, essere ridotta ad una sola dimensione.

$\mathrm{Ci}$ si potrebbe perciò attendere che coloro i quali hanno un approccio strumentale al voto, cioè coloro che guardano all'atto del votare in termini di interesse personale, siano più facilmente attratti da una partecipazione politica monotematica rispetto a coloro che intendono il voto come una virtù civica. Invece, contrariamente alle aspettative, non c'è differenza nella propensione alla partecipazione monotematica tra approccio al voto come dovere civico e approccio utilitaristico (vedi TAB. 6).

TAB. 6. - Percentuale di coloro che considerano il voto un dovere civico oppure una questione di interesse personale in funzione di un indice di partecipazione politica basato sulla presenza a manifestazioni elo sulla firma di petizioni.

\begin{tabular}{lcccc}
\hline & 0 & 1 & 2 & $\mathrm{~N}$ \\
& (nessuno) & (l'uno o l'altro) & (entrambi) & 2731 \\
\hline Dovere civico & 65 & 27 & 8 & 376 \\
\hline
\end{tabular}

Fonte: Sondaggi Elezioni Amministrative 1999.

Una prima e più intuitiva interpretazione ci spinge ad affermare che questa invarianza sia dovuta al fatto che le varie istanze sulle quali si ha mobilitazione coprono un ampio spettro di tematiche. Di conseguenza, su alcune di esse, i partecipanti sono spinti dall'interesse personale e su altre, da considerazioni altruistiche 
o più legate al dovere civico. Una diversità di valori, ossia interesse personale o dovere civico, rispetto alla concezione dell'atto di recarsi alle urne, non è dunque rilevante al fine di interpretare la partecipazione politica monotematica.

\section{Ricapitolazione}

Il crollo dell'affluenza alle urne alle elezioni locali in Norvegia ha comportato l'emergere di un nuovo modello di partecipazione elettorale. Gli uomini delle città erano la categoria con la più alta propensione al voto, le donne della provincia quella con la propensione più bassa. Adesso il quadro sembra essere l'opposto. Ciò significa che il calo della partecipazione elettorale è stato più marcato tra gli uomini nelle aree più densamente popolate.

Tale declino è stato analizzato in questo saggio alla luce di alcuni aspetti, in particolare soffermandosi su alcune sue cause ed alcune sue conseguenze. In seguito si è discusso dell'incremento della partecipazione politica monotematica.

Rispetto alle conseguenze, è stata esclusa quella riguardante il calo della fiducia nel sistema politico che si è visto non essere valida nel caso norvegese. È stata poi considerata quella che prevede, a seguito del declino della partecipazione elettorale, la penalizzazione dei partiti di sinistra. Tuttavia la tesi secondo cui, appunto, il partito laburista norvegese avrebbe sofferto elettoralmente di tale declino non è stata confermata. Dobbiamo però ammettere che la nostra base empirica non è solida.

Per quanto riguarda le cause, la discussione si è concentrata su due approcci valoriali al voto: quello strumentale-utilitaristico, che sottolinea l'interesse personale come fattore trainante, e quello normativo, che ha invece come impulso determinante il dovere civico.

Un punto centrale dell'approccio strumentale è il livello d'importanza attribuito all'elezione in questione. Si poteva supporre che un calo della partecipazione dovesse essere seguito da un'opinione diffusa che considerasse le elezioni amministrative sempre più irrilevanti. Ed invece è stato riscontrato esattamente il contrario. Infatti, nel 1971 la quota di elettori che sosteneva che l'elezione aveva un basso impatto, o un impatto nullo, su quello che sarebbe accaduto nella politica comunale nei quattro anni successivi, era più ampia rispetto al 1999, quando il tasso di partecipazione elettorale è stato invece molto inferiore.

Particolarmente pronunciato è stato il crollo della partecipazione tra coloro che considerano le elezioni irrilevanti. Questo è un indicatore del fatto che l'idea del voto come virtù civica si sta affievolendo. Infatti, per coloro che ritengono il voto un dovere civico, ogni cittadino sarebbe obbligato a recarsi alle urne a prescindere dall'importanza dell'elezione in questione. Purtroppo la domanda relativa al dovere civico è stata posta soltanto nella ricerca sulle elezioni del $1999 \mathrm{e}$, di conseguenza, non abbiamo serie temporali di dati.

É stato poi disaggregato per coorti, sempre con i dati relativi alle amministrative del 1999, il tasso di partecipazione elettorale di coloro che mutuano l'ap- 
proccio utilitaristico al voto e che quindi ritengono che la gente dovrebbe votare solo se l'elezione riguarda direttamente i suoi interessi personali. La differenza tra le classi di età è marcata. Nella coorte dei giovanissimi è più frequente trovare l'opzione del voto come interesse personale rispetto alle generazioni precedenti.

Anche la propensione a recarsi alle urne è stata analizzata per coorti ed è stato rilevato come rispetto ad essa le differenze intergenerazionali siano aumentate nel tempo. Tra i giovanissimi il tasso di partecipazione elettorale del 1999 è stato drammaticamente basso. Siamo stati perciò tentati di mettere in relazione questo dato con un cambiamento nei valori riguardanti l'atto di votare e, quindi, di concludere che una visione strumentale del voto, oggi più diffusa rispetto ad una fondata sul dovere civico, è una causa del calo generale del tasso di partecipazione elettorale.

É però altrettanto vero che i giovani non mostrano passività politica in senso assoluto. In attività politiche focalizzate su tematiche specifiche, dove a differenza dell'affluenza alle urne si è registrato un andamento in crescita della partecipazione, i giovani sono la categoria più attiva. Inoltre, la coorte dei giovanissimi, cioè dei nati dopo il 1975, devia rispetto all'andamento generale dal momento che i non votanti di questa categoria, sempre rispetto alla partecipazione monotematica, sono più attivi dei votanti. Il coinvolgimento politico è dunque incanalato in direzioni alternative rispetto all'arena elettorale. Ciò può essere interpretato anche in termini di ciclo di vita e ciò significa che all'aumentare dell'età anagrafica il modello di comportamento probabilmente cambierà.

Pertanto, se è vero che l'interpretazione generazionale è la più appropriata per questi fenomeni, ciò significa che il calo della partecipazione elettorale, così come l'aumento della partecipazione monotematica, sono destinati a continuare nel tempo.

Per concludere, vogliamo sollevare un interrogativo di ricerca: perché la crisi del voto come dovere civico non ha penalizzato il tasso di partecipazione alle elezioni politiche quanto alle amministrative?

Come possiamo vedere ancora dalla FIG. 1, la forbice tra il tasso di partecipazione alle elezioni locali e quello alle parlamentari si è progressivamente allargata. Una possibile spiegazione è che le elezioni politiche siano considerate molto più importanti di quelle amministrative ${ }^{10}$. Dal momento che si affievolisce l'idea del voto come dovere civico, diventa sempre più cruciale il fattore "rilevanza dell'elezione". Il fatto che la partecipazione alle elezioni amministrative, a differenza delle politiche, sia più alta nei comuni scarsamente popolati, può essere spiegato dal fatto che le elezioni locali sono considerate tanto più importanti quanto minore è il numero di abitanti del comune stesso. In effetti, le elezioni politiche sono percepite come più importanti rispetto alle amministrative, ma ci sono delle

\footnotetext{
${ }^{10}$ Nella ricerca sulle elezioni locali del 1995 fu posta la seguente domanda: «Quali elezioni Lei considera più importanti: quelle nazionali, quelle di contea o quelle comunali?». Il $71 \%$ rispose quelle nazionali, l'1\% quelle di contea ed il $28 \%$ le elezioni comunali.
} 
differenze in funzione della grandezza del comune. Nel 1995, nei comuni con meno di 2.500 abitanti, la differenza tra coloro che ritenevano più rilevanti le elezioni a livello nazionale rispetto a coloro che invece valutavano di più le amministrative era di 11 punti percentuali a favore delle elezioni politiche. Nei comuni con più di 60.000 abitanti la differenza, sempre a favore delle elezioni politiche, saliva invece al $61 \%$.

Una delle ragioni che più frequentemente vengono addotte per spiegare l'irrilevanza delle elezioni amministrative in Norvegia è la ristrettezza dello spazio di manovra politica dei comuni, dal momento che gli enti locali sarebbero gli esecutori di una linea politica che è, in realtà, diretta dal centro. In effetti, anche alcuni studi sulle priorità economiche dei comuni norvegesi tra i diversi settori (amministrazione, istruzione, servizi sociali, ecc...) rivelano differenze minime rispetto al colore politico del consiglio comunale.

In questo contesto Rune Sørensen (1989) conclude, pur ponendo un punto interrogativo alla propria affermazione, che votare alle elezioni amministrative è «un'espressione simbolica di cittadinanza politica». Tuttavia, come abbiamo visto, molti elettori norvegesi hanno una diversa opinione e ritengono che le elezioni locali siano importanti, sia rispetto alle loro convinzioni politiche, sia rispetto al futuro dell'ente locale. 


\section{Riferimenti bibliografici}

AARDAL B. (1999), «Politikerforakt og politisk mistillit», in AARDAL B. et al., Velgere i 90-årene, Oslo, NKS-Forlaget.

BALDERSHEIM H., ROSE L.E. (2000), «Kommunale reformer som teoretiske utfordringer: Linjer og paradokser i lokal politikk og organisering», in BALDERSHEIM H., RosE L.E. (a cura di), Det kommunale laboratorium. Teoretiske perspektiver på lokal poltikk og organisering, Bergen, Fagbokforlaget.

BJØRKLUND T. (1999), Et lokalvalg $i$ perspektiv. Valget $i 1995$ i lys av sosiale og politiske endringer, Oslo, Tano-Aschehoug.

BjØRKLUND T., SAGLIE J. (2000), Lokalvalget i 1999. Rekordlav og rekordbøy deltakelse, Rapporto del 12/2000, Oslo, Institutt for samfunnsforskning.

BLAIS A., YOUNG R., LAPP M. (2000), «The calculus of voting: An empirical test», in European Fournal of Political Research, 37, pp. $181-201$.

Downs A. (1957), An economic theory of democracy, New York, Harper and Row.

FraNKLIN M.N. (1996), «Electoral Participation», in LEDUC L., NIEMI R.G., NORRIS P. (a cura di), Comparing Democracies. Elections and Voting in Global Perspective, London, Sage.

Goul ANDERSEN J., Hoff J. (2000), Democracy and Citizenship in the Scandinavian Welfare State, Hampshire, Macmillan (forthcoming).

HofFMANN-MARTINOT V. (1994), «Voter turnout in French municipal elections», in LOPEZ NIETO L. (a cura di), Local Elections in Europe, Barcelona, ICPS. KirChHeIMER O. (1966), «The Transformation of Western European Party System», in LaPalombara J., Weiner M. (a cura di), Political Parties and Political Development, New Haven, Yale University Press.

KeY V.O. Jr (1958), Politics, Parties and Pressure Groups, New York, Crowell.

LijPHART A. (1997), «Unequal Participation: Democracy's Unresolved Dilemma», in American Political Science Review, 91, pp. 1-14.

Listhaug O., Wiberg M. (1995), «Confidence in Political and Private Institutions», in Klingemann H.-D., Fuchs D. (a cura di), Citizens and the State, Oxford, Oxford University Press.

Miller W.E. (1992), «The Puzzle Transformed: Explaining Declining Turnout», in Political Behavior, 14, pp. 1-43.

OlsEn J. P., SÆTREN H. (1980), Aksjoner og demokrati, Bergen, Universitetsforlaget.

PACEK A., RADCLIFF B. (1995), «Turnout and the Vote for Left-of-Centre Parties: A Cross-National Analysis», in British Journal of Political Science, 25, pp. 137143.

ROKKan S., VALEN H. (1962), «The Mobilization of the Periphery: Data on Turnout, Party Membership and Candidate Recruitment in Norway», in Acta Sociologica, 6, pp. 111-158 (pubblicato anche in ROKKAN S. (1970), Citizens, Elections, Parties, Oslo, Universitetsforlaget, trad.it., Cittadini, elezioni, partiti, Il Mulino, Bologna, 1982). 
RosE R. (1997), «Evaluating Election Turnout», in Voter Turnout from 1945 to 1997: a Global Report on Political Participation, pubblicato dall'International Institute for Democracy and Electoral Assistance, IDEA, Stoccolma.

Rosenstone S., Hansen J.M. (1993), Mobilization, Participation, and Democracy in America, New York, Macmillan.

SøRENSEN R. (1989), «Det kommunale demokrati: En fortsettelse av rikspolitikken med andre midler?», in Norsk Statsvitenskapelig Tidsskrift, 4, pp. 267 - 292. TOPF R. (1995a), «Electoral Participation», in KLINGEMANN H.-D., FUCHS D. (a cura di), Citizens and the State, Oxford, Oxford University Press.

Topf, R. (1995b), «Beyond Electoral Participation», in KLINGEManN H.-D., FuCHS D. (a cura di), Citizens and the State, Oxford, Oxford University Press. 